\title{
USO DE MESOCARPO DE MARACUJÁ COMO BIOSSORVENTE DE Cr(VI)
}

\author{
D. D. A. NUNES ${ }^{1}$, J. C. T. REZENDE ${ }^{1}$, E. N. dos REIS ${ }^{1}$, E. de JESUS ${ }^{1}$, A. S. SILVA ${ }^{2}$, R. L. \\ PAGANO $^{1}$ \\ ${ }^{1}$ Universidade Federal de Sergipe, Departamento de Engenharia Química \\ ${ }^{2}$ Universidade Federal de Sergipe, Departamento de Matemática \\ E-mail para contato: denner.deda@hotmail.com
}

\begin{abstract}
RESUMO - Este trabalho teve como objetivo avaliar o potencial de utilização do mesocarpo de maracujá (Passiflora edulis) na adsorção de $\mathrm{Cr}(\mathrm{VI})$ de soluções aquosas. Na obtenção do biomaterial, o mesocarpo do maracujá passou por tratamento térmico a $400{ }^{\circ} \mathrm{C}$, seguido de trituração e classificação granulométrica. A determinação de $\mathrm{Cr}(\mathrm{VI})$ foi feita por espectrofotometria UV-visível através da complexação com 1,5-difenilcarbazida, medindo a absorbância no comprimento onda $540 \mathrm{~nm}$. Foram realizados experimentos variando o tempo de contato e o $\mathrm{pH}$ inicial. A eficiência da remoção de íons de $\mathrm{Cr}(\mathrm{VI})$ foi avaliada por espectroscopia de infravermelho. A remoção média do biomaterial utilizado foi de $68 \%$.
\end{abstract}

\section{INTRODUÇÃO}

A atividade humana, em seus múltiplos aspectos, se reflete em uma crescente diversidade e contínua produção de resíduos, sendo necessário assegurar a sua destinação adequada para minimizar os impactos negativos sobre a saúde e meio ambientes (BERNARDO, 2011). As indústrias a exemplo das metalúrgicas e químicas produzem grandes quantidades de metais pesados em águas residuais a cada ano. Parte disso é derramada em corpos d'água sem tratamento adequado ou sem tratamento, o que resulta em poluição do ambiente aquático, pois os metais pesados não são eliminados do meio ambiente (XU e FANG, 2006).

$\mathrm{O} \mathrm{Cr}(\mathrm{VI})$ é geralmente produzido por processos industriais, sendo resultante de processos como cromagem, fabricação de corantes e pigmentos, curtimento de couro, madeira e preservação (RUSSEL, 2004). A toxicidade do cromo para a vida aquática é dependente de fatores como a temperatura, $\mathrm{pH}$, espécie, estado de oxidação e concentração de oxigênio (KLAASSEN e WATKINS, 2001). O Cr(III) é essencial do ponto de vista nutricional, não tóxico e pobremente absorvido no organismo, enquanto o cromo hexavalente afeta os rins e o sistema respiratório (HELLER e PÁDUA, 2010).

Muitos métodos físico-químicos foram desenvolvidos para a remoção de metais pesados de soluções aquosas, como a extração, troca iônica, precipitação química e processos de separação por membrana. Esses métodos têm diversas desvantagens como altos custos operacionais, baixa seletividade, remoção incompleta, e produção de grande quantidade de rejeitos. Outro método popular para a remoção de metais pesados de soluções aquosas é a 
adsorção. Parâmetros têm que ser levados em consideração na escolha de adsorventes apropriados, como: a capacidade de adsorção, habilidade de reutilização, parâmetros cinéticos, preço e mercados disponíveis. Rejeitos de vários processos industriais e materiais biológicos - biossorventes - são também usados. A biossorção é a capacidade natural da biomassa que imobiliza componentes dissolvidos, íons de metais pesados, na sua superfície. A biomassa é composta em sua maioria por polissacarídeos, proteínas e gorduras, e tem muitos grupos funcionais que são capazes de capturar íons metálicos (WITEK-KROWIAK et al., 2011).

Este trabalho tem como finalidade estudar a capacidade de utilização do mesocarpo de maracujá como biossorvente na remoção de $\mathrm{Cr}(\mathrm{VI})$ de soluções aquosas visando contribuir com um novo método alternativo para ser aplicado na recuperação de águas contaminadas com $\mathrm{Cr}(\mathrm{VI})$.

\section{MATERIAIS E MÉTODOS}

\subsection{Preparação do Biossorvente}

O mesocarpo de frutos de maracujá foi removido e finamente dividido. Em seguida passou por tratamento térmico em mufla (GP Científica) a $400^{\circ} \mathrm{C}$ durante 20 minutos. Em seguida, o material foi triturado.

\subsection{Determinação do Cromo(VI)}

A solução estoque de $\mathrm{Cr}(\mathrm{VI})$ foi preparada a partir de dicromato de potássio (Merk) dissolvendo 2,829 g em $1000 \mathrm{~mL}$ de água destilada, formando assim, uma solução de 1000 $\mathrm{mgL}^{-1}$ de $\mathrm{Cr}(\mathrm{VI})$. Foram obtidas, por diluições, soluções nas concentrações padrões de 0,2 ; 0,4; 0,8 e 1,0 mg. $\mathrm{L}^{-1}$. Segundo Morita e Assumpção (2007) a cor roxo-violeta desenvolvida na reação entre $\mathrm{Cr}(\mathrm{VI})$ e 1,5-difenilcarbazida em meio ácido. Para as análises de $\mathrm{Cr}(\mathrm{VI})$ foram utilizados $25 \mathrm{~mL}$ de ácido sulfúrico (IMP) $0,01 \mathrm{molL}^{-1}$ e $2 \mathrm{~mL}$ de complexante 1,5difenilcarbazida, alíquota da solução a ser analisada e completado o volume de $100 \mathrm{~mL}$ com água destilada. A absorbância foi medida no espectrofotômetro (UV-VIS da VARIAN) no comprimento de onda de $540 \mathrm{~nm}$. As concentrações de $\mathrm{Cr}(\mathrm{VI})$ desconhecidas após o início da adsorção foram determinadas usando o valor da absorbância da amostra. A porcentagem de $\mathrm{Cr}(\mathrm{VI})$ removida foi determinada pela Equação 1.

$$
\% \text { Remoçãode Cr(VI) }=\left[\frac{\left(C_{i}-C_{f}\right)}{C_{i}}\right] \times 100
$$




\subsection{Espectroscopia de Absorção na Região do Infravermelho}

A espectroscopia de infravermelho foi realizada no biossorvente antes e depois da adsorção. As análise foram realizadas utilizando espectrômetro modelo iS-10 FT-IR da Nicolet e utilizando pastilhas de $\mathrm{KBr}$.

\subsection{Efeito do pH}

No estudo do efeito do pH na adsorção do biomaterial foi utilizada solução 5 ppm de $\mathrm{Cr}(\mathrm{VI}) ; 0,1 \mathrm{~g}$ do biossorvente, $\mathrm{pH}$ inicial de 2, 5, 7 e 8; tempo de contato foi de 120 minutos. Os testes em batelada foram realizados em mesa orbital Nova Ética modelo 109 a 150 rpm.

\subsection{Efeito do Tempo de Contato}

No estudo do efeito do tempo de contado foi utilizada solução de $\mathrm{Cr}(\mathrm{VI})$ a 5 ppm; $0,1 \mathrm{~g}$ de biossorvente, $\mathrm{pH}$ inicial 2, velocidade de $150 \mathrm{rpm}$. O tempo variou de 10 a $140 \mathrm{~min}$.

\subsection{Cinética de Adsorção}

O estudo cinético é importante para identificar a forma que ocorre a transferência de massa do adsorbato para o adsorvente. Os modelos de pseudoprimeira ordem e de pseudosegunda ordem são geralmente utilizados nesta análise. O modelo pseudoprimeira ordem é representado pela Equação 2:

$$
\frac{\mathrm{dQ}_{\mathrm{t}}}{\mathrm{dt}}=\mathrm{k}_{1}\left(\mathrm{Q}_{\mathrm{e}}-Q_{t}\right)
$$

em que $\mathrm{k}_{1}$ é a constante de adsorção de pseudoprimeira ordem $\left(\min ^{-1}\right), \mathrm{Q}_{\mathrm{e}} \mathrm{e} \mathrm{Q}_{\mathrm{t}}$ são as quantidades adsorvidas por grama de biossorvente no equilíbrio e no tempo $t$, respectivamente $\left(\mathrm{mmols} \mathrm{g}^{-1}\right)$. Integrando a Equação $1 \mathrm{de} \mathrm{Q}_{\mathrm{t}}=0 \mathrm{em} \mathrm{t=0} \mathrm{a} \mathrm{Q}_{\mathrm{t}}=\mathrm{Q}_{\mathrm{e}} \mathrm{em} \mathrm{t}=\mathrm{t}$ obtém-se a Equação 3:

$$
\ln \left(Q_{e}-Q_{t}\right)=\ln Q_{e}-k_{1} t
$$

o valor de $k_{1}$ e de $Q_{e}$ não experimental podem ser obtidos do gráfico $\ln \left(Q_{\mathrm{e}}-\mathrm{Q}_{\mathrm{t}}\right)$ versus $\mathrm{t}$.

O modelo pseudosegunda ordem é descrito pela Equação 4: 


$$
\frac{\mathrm{dQ}_{\mathrm{t}}}{\mathrm{dt}}=\mathrm{k}_{2}\left(\mathrm{Q}_{\mathrm{e}}-Q_{t}\right)^{2}
$$

em que $\mathrm{k}_{2}$ é a constante da taxa de adsorção de pseudosegunda ordem $\left(\mathrm{g} \mathrm{mg}^{-1} \mathrm{~min}^{-1}\right)$.

Integrando a Equação 4 nos moldes da Equação 2 e linearizado-a tem-se a Equação 5:

$$
\frac{\mathrm{t}}{Q_{t}}=\frac{1}{k_{2} Q_{e}^{2}}+\frac{1}{\mathrm{Q}_{\mathrm{e}}}
$$

O modelo intrapartícula de Weber e Moris (Equação 6) fornece informações sobre o fator determinante da velocidade de adsorção, sendo $\mathrm{Q}_{\mathrm{t}}$ a quantidade de $\mathrm{Cr}(\mathrm{VI})$ adsorvida de (mmols $\left.\mathrm{g}^{-1}\right), \mathrm{t}$ o tempo de adsorção (min), $\mathrm{C}$ (mmols $\mathrm{g}^{-1}$ ) uma constante relacionada coma resistência à difusão intrapartícula e $\mathrm{k}_{\text {dif }}$ é o coeficiente de difusão intrapartícula (mmols $\mathrm{g}^{-1}$ $\min ^{-1 / 2}$ ). Este modelo possibilita identificar os mecanismos de transferência de massa envolvidos no processo de adsorção.

$$
\mathrm{Q}_{\mathrm{t}}=\mathrm{k}_{\mathrm{dif}} \mathrm{t}^{\frac{1}{2}}+C
$$

\section{RESULTADOS E DISCUSSÕES}

A remoção de $\mathrm{Cr}(\mathrm{VI})$ foi melhor no $\mathrm{pH} 2$ com remoção de $68 \%$; estudos como o de Pehlivan et al. (2012) e Altun (2012) e Pehlivan (2012) também apontam tal pH como o ótimo para a adsorção de $\mathrm{Cr}(\mathrm{VI})$. A variação de $\mathrm{pH}$ provoca mudanças nas características das espécies de $\mathrm{Cr}(\mathrm{VI})$ e na superfície do biomaterial. A baixa remoção em $\mathrm{pH}$ inicial elevado pode ser em decorrência da concorrência entre $\mathrm{OH}$ e as espécies de $\mathrm{Cr}(\mathrm{VI})$ pela superfície do biossorvente (CONCEICAO, 2014).

A Figura 1 mostra a quantidade de remoção de $\mathrm{Cr}(\mathrm{VI})$ adsorvida com tempo. A quantidade removida é crescente até o momento em que o biossorvente e o meio entram em equilíbrio de adsorção. O tempo de equilíbrio encontrado para este experimento foi de 120 minutos após o inicio da adsorção. O equilíbrio na remoção de $\mathrm{Cr}(\mathrm{VI})$ pode ser explicado pela utilização de todo o sítio ativo do pó de maracujá. Durante a adsorção, o $\operatorname{Cr}(\mathrm{VI})$ se impregna na superfície do adsorvente até que toda a superfície esteja coberta de íons, neste ponto a concentração de $\mathrm{Cr}(\mathrm{VI})$ deixa de diminuir e se mantém em equilíbrio com o adsorvente em solução, com isso, o tempo de equilíbrio da adsorção varia de acordo com a superfície do adsorvente utilizado (RAMOS, 2013). 


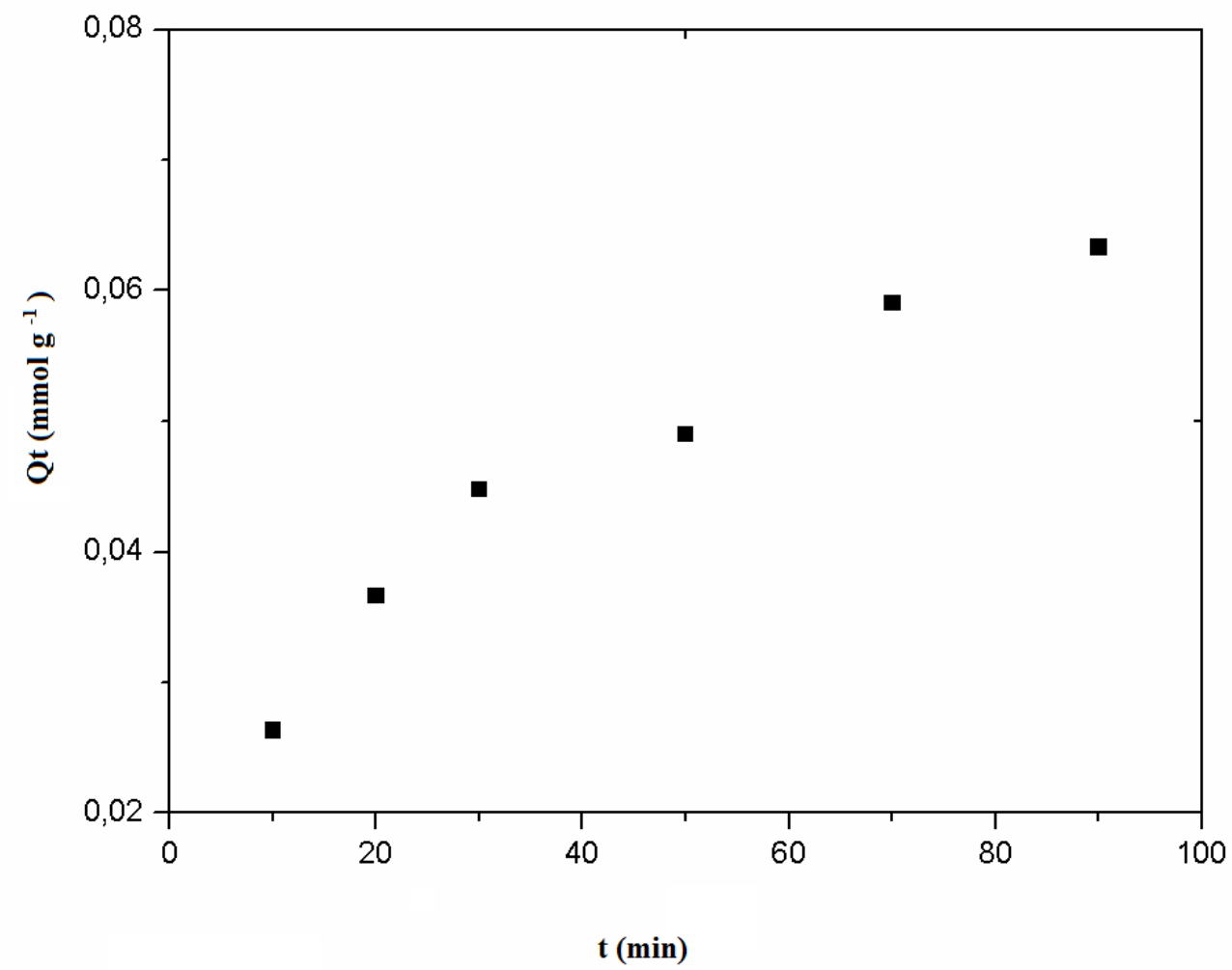

Figura 1. Efeito do tempo de contato na remoção de $\mathrm{Cr}(\mathrm{VI})$. Condições: concentração inicial de $\mathrm{Cr}(\mathrm{VI}) 5 \mathrm{mg} \mathrm{L}^{-1}$; volume do meio, $100 \mathrm{~mL}$; quantidade de adsorvente, $0,1 \mathrm{~g}$; temperatura $25 \pm 1{ }^{\circ} \mathrm{C}$; velocidade de agitação de $150 \mathrm{rpm}$; $\mathrm{pH} 2$ para o pó do mesocarpo de maracujá.

A análise FTIR do biossorvente antes e depois da adsorção é mostrada na Figura 2. A banda larga e forte em $3400 \mathrm{~cm}^{-1}$ foi atribuída ao grupo hidroxila (-OH) de ácidos carboxílicos (PEHLIVAN et al., 2012). O pico em $2922 \mathrm{~cm}^{-1}$ indica a vibração do C-H do grupo $-\mathrm{CH}_{2}$ (PEHLIVAN et al., 2012). A banda de aproximadamente $1600 \mathrm{~cm}^{-1}$ foi atribuída à vibração da carbonila $(\mathrm{C}=\mathrm{O})$ de ácidos carboxílicos (BARBOSA, 2008) A banda em $1380 \mathrm{~cm}^{-1}$ pode ser referida à ligação N-O em compostos alifáticos. Observando os espectros percebe-se que houve uma mudança na transmitância dos grupos identificados, pois o $\mathrm{Cr}(\mathrm{VI})$ ao interagir com estes grupos provavelmente modifica a capacidade de absorção de energia dos mesmos. 


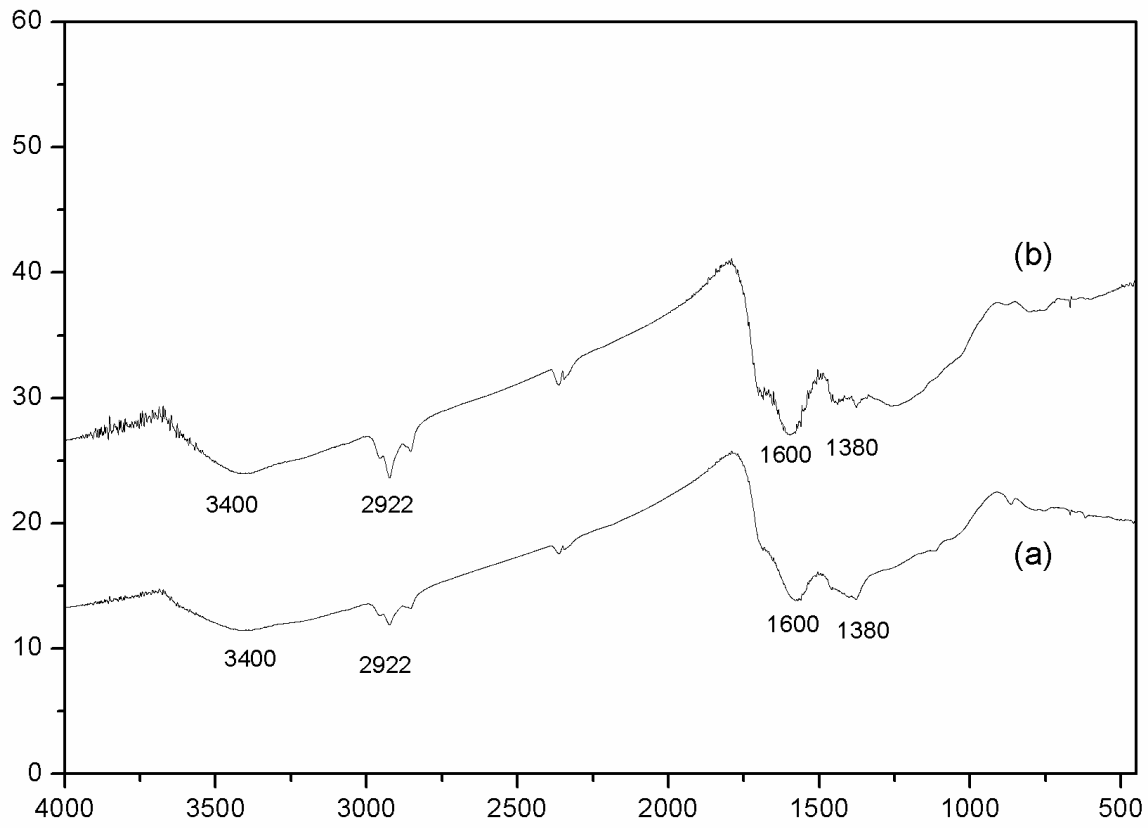

Figura 2 - Análise de Espectroscopia de Absorção para o biossorvente. O (a) representa $\mathrm{o}$ antes do processo adsortivo e o (b) representa o depois do mesmo processo.

No estudo cinético, verificou-se que o melhor ajuste foi utilizando o modelo pseudosegunda ordem (Figura 3), em que se obteve $\mathrm{R}^{2}=0,99$, valor de $\mathrm{k}_{2}$ de $0,5634 \mathrm{~g} \mathrm{mmols}^{-1} \mathrm{~min}^{-1}$ e $\mathrm{Q}_{\mathrm{e}}$ calculado de 0,0770 mmols $\mathrm{g}^{-1}$. Utilizando o modelo de adsorção de Weber e Moris obteve-se $\mathrm{R}^{2}$ igual a $0,9800, \mathrm{k}_{\mathrm{dif}}$ de $0,0057 \mathrm{mmols} \mathrm{g}^{-1} \mathrm{~min}^{-1 / 2}$ e C igual a $0,0104 \mathrm{mmols} \mathrm{g}^{-1}$. Como o valor de $\mathrm{C}$ foi próximo de zero e os dados experimentais se ajustaram ao modelo intrapartícula de Weber e Morris, sugere-se que o mecanismo de transferência de massa durante a adsorção do $\mathrm{Cr}(\mathrm{VI})$ provavelmente é tanto por mecanismo intrapartícula como mecanismo de formação de camada limite externa (ROCHA et al., 2012). 


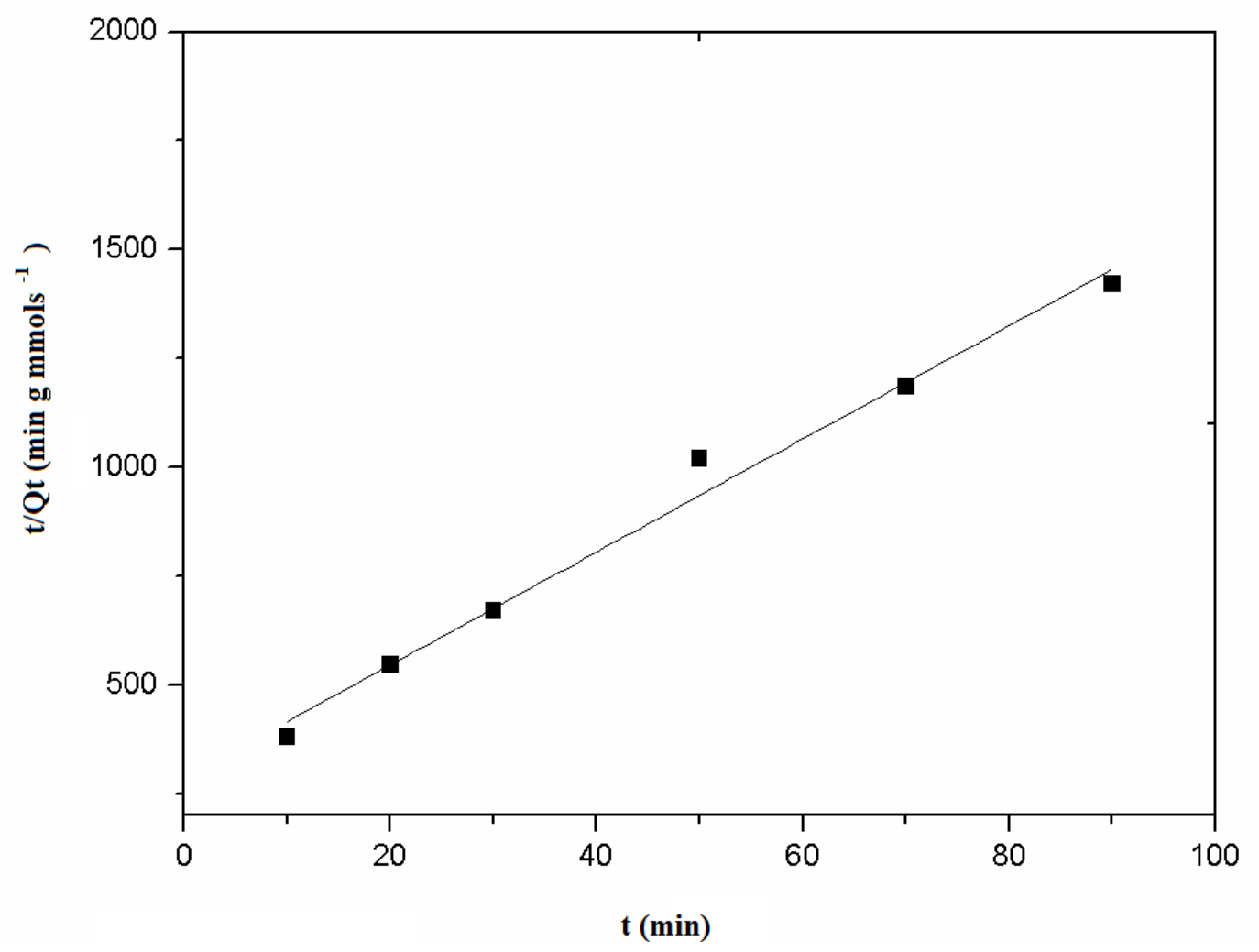

Figura 3: Cinética de adsorção pseudosegunda ordem (Equação 5).

\section{CONCLUSÃO}

O tempo de equilíbrio encontrado foi de 120 minutos e a porcentagem de remoção encontrada foi de aproximadamente $68 \%$ neste tempo no $\mathrm{pH}$. Considerando os resultados apresentados, pode ser concluído que a remoção de $\mathrm{Cr}(\mathrm{VI})$ aumenta com o aumento do tempo de contato entre adsorvente e adsorvato até atingir um tempo de equilíbrio. A porcentagem de remoção do íon depende do tempo de contato e pH. A maior remoção de $\mathrm{Cr}(\mathrm{VI})$ foi de $68 \%$ utilizando $0,1 \mathrm{~g}$ de biossorvente com $\mathrm{pH}$ inicial igual a 2. Verificou-se uma cinética rápida, sendo que em 120 min foi atingido equilíbrio. A transferência de massa durante a adsorção foi dominada pelo fator difusional intrapartícula como pela formação de camada limite externa. Sendo assim, outros estudos estão sendo desenvolvidos para se determinar a capacidade de adsorção do mesocarpo de maracujá tratado termicamente na remoção de Cr(VI).

\section{REFERÊNCIAS}

ALTUN, T.; PEHLIVAN, E. Removal of Cr(VI) from aqueous solutions by modified walnut shells. Food Chemistry., v. 132, p. 693-700, 2012

BARBOSA, L. C. A.; Espectroscopia no infravermelho na caracterização de compostos orgânicos. Editora UFV, $1^{\circ}$ reimpressão. Viçosa, MG: UFV, 2008. 
BERNARDO, M. M. S. Physico-chemical characterization of chars produced in the copyrolysis of wastes and possible routes of valorization. Tese de Mestrado, 2011.

CONCEICAO, J. C.; RAMOS, V. H. S.; JESUS, E.; SILVA, A. S.; COSTA, A. W. M. C. C. Biosortion of $\mathrm{Cr}(\mathrm{VI})$ from Aqueous Solutions using Chemically Modified Okra Powder. Journal of Basic \& Applied Sciences., v.10, p. 73-79, 2014.

DERMIBAS, A. Agricultural based activated carbons for the removal of dyes from aqueous solutions: a review, Jornal of Hazardous Materials., v.167, p. 1-9, 2009.

DING, Y.; JING, D.; GONG, H.; ZHOU; YANG, X.; Biosorption of aquatic cadmium(II) by unmodified rice straw. Bioresource Technology., v. 114, p. 20-25, 2012.

FRANCA, A. S.; OLIVEIRA, L. S.; FERREIRA, M. E. Kinetics and equilibrium studies of methylene blue adsorption by spent coffee grounds. Desalination., v.249, p. 267-272, 2009.

GONZALES, M. H.; ARAUJO, G. C. L.; PELIZARO, C. B.; MENEZES, E. A.; LEMOS, S. G.; SOUSA, G. B.; NOGUEIRA, A. R. A.; Coconut coir as biosorbent for $\mathrm{Cr}(\mathrm{VI})$ removal from laboratory wastewater. Journal of Hazardous Materials., v.159, p. 252256, 2008.

HELlER, L.; PÁDUA, V. L.; Abastecimento de água para consumo humano. Belo Horizonte: Editora UFMG, 2010.

HOSSAIN, M. A.; NGO, H. H.; GUO W. S.; NGUYEN, T. V.; Biosorption of Cu(II) From Water by Banana Peel Based Biosorbent: Experiments and Models of Adsorption and Desorption. Journal of Water Sustainability., v. 2, p. 87-104, 2012.

KLAASSEN, C. D.; WATKINS, J. B. Casarett \& Doull, Manual de toxicologia. México: Editora Mc Graw-Hill Interamericana., 2001.

MORITA, T.; ASSUMPÇÃO, R. M. V. Manual de soluções, reagentes e solventes: padronização, preparação, purificação com indicadores de segurança e de descarte de produtos químicos. São Paulo: Editora Blucher, 2007.

PEHLIVAN, E.; PEHLIVAN, E.; TUTAR, H. K. Hexavalent chromium removal by Osage Orange. Food Chemistry., v. 133, p 1478-1484, 2012.

PINO, G. A. H.; MESQUITA, L. M. S.; TOREM, M. L.; Biossorção de Metais Pesados Utilizando Pó da Casca de Coco Verde (Cocos nucifera). Dissertação de Mestrado, 2005.

RAMOS, V. H. S.; CONCEICAO, J. C.; JESUS, E.; SILVA, A. S.; SILVA, D. C. Remoção de cromo hexavalente por biossorção usando pó de quiabo. XXXVI Congresso Brasileiro de Sistemas Particulados, 2013.

ROCHA, O. R. S.; NASCIMENTO, G. E.; CAMPOS, N. F.; SILVA, V. L.; DUARTE, M. M. M. B. Avaliação do processo adsortivo utilizando mesocarpo de coco verde para remoção do corante cinza reativo BF-2R. Química Nova., vol. 35, No. 7, p. 1369-1374, 2012

RUSSEL, J.B. Química Geral.., 2 ${ }^{\text {a }}$ Ed ,Vol 1, Editora : Pearson Education, 2004.

WITEK-KROWIAK, A.; SZAFRAN, R. G.; MODELSKI, S. Biosorption of heavy metals from aqueous solutions onto peanut shell as a low-cost biosorbent. Desalination., v. 265,p.126-134, 2011.

XU,Y.; FANG, Z. Experimental research on heavy metal wastewater treatment with dipropyl dithiophospate. Journal of Hazardous Materials.,v. B137, p. 1636-1642, 2006. 